\title{
Development of Quadruple-ridge Flared Horn with Spline-defined Profile for Band B of the Wide Band Single Pixel Feed (WBSPF) Advanced Instrumentation Programme for SKA
}

\author{
Jian Yang \\ Dept. of Signals and Systems \\ Chalmers University of Technology, Sweden \\ jian.yang@chalmers.se
}

\author{
Jonas Flygare, Miroslav Pantaleev and Bhushan Billade \\ Onsala Space Observatory, \\ Chalmers University of Technology, Sweden \\ jonas.flygare, miroslav.pantaleev, bhushan.billade@chalmers.se
}

\begin{abstract}
A new quadruple-ridge flared horn with numerically defined profiles by spline function is proposed for Band $B$ of the Wide Band Single Pixel Feed (WBSPF) Advanced Instrumentation Programme for SKA in the paper. Optimization for high aperture efficiency on the spline-profiles have been carried out, resulting in that the aperture efficiency is higher than $65 \%$ over 4.6-20 $\mathrm{GHz}$ and reflection coefficient is below $-10 \mathrm{~dB}$.
\end{abstract}

\section{INTRODUCTION}

The Square Kilometre Array (SKA) [1] is an interferometric radio telescope infrastructure designed to have a combined collecting area of one square kilometres. In its final form this telescope will be 50 times more sensitive than the present largest radio interferometer and will survey the sky 10,000 times faster. It will tackle key scientific questions, such as mapping the cosmic epoch of reionization (when the first galaxies and stars formed), tracing the evolution of gas content and magnetic fields in galaxies through cosmic time, etc. Pulsars will be utilised to test theories of gravity and to detect very low frequency gravitational waves. This project has entered its pre-construction (final design) phase which covers the period 2014-2016. Sweden (represented by Onsala Space Observatory, Chalmers) is a member of the SKA Organisation and participates in two of its design consortia. One important task for us is to complete during 2015/2016 the committed technical work for wide-band feed design for reflectors.

The challenges in this work include the following: 1) high aperture efficiency, reaching above $78 \%, 70 \%, 65 \%$ and $60 \%$ over 4.6-8, 8-15, 15-20 and 20-24 GHz, respectively; 2) high sensitivity which requires both high aperture efficiency and low noise temperature; 3) good impedance matching between the feed and LNAs in order to reduce the noise temperature; 4) high radiation efficiency (low ohmic loss of the feed) in order to reduce the system noise temperature; 5) a compact size but without very tiny parts in the feed so that the whole feed can be inside a compact cryostat and the manufacture can be done at low cost.

This paper reports the development of a quadruple-ridge flared horn (QRFH) with spline-defined profile as a feed for

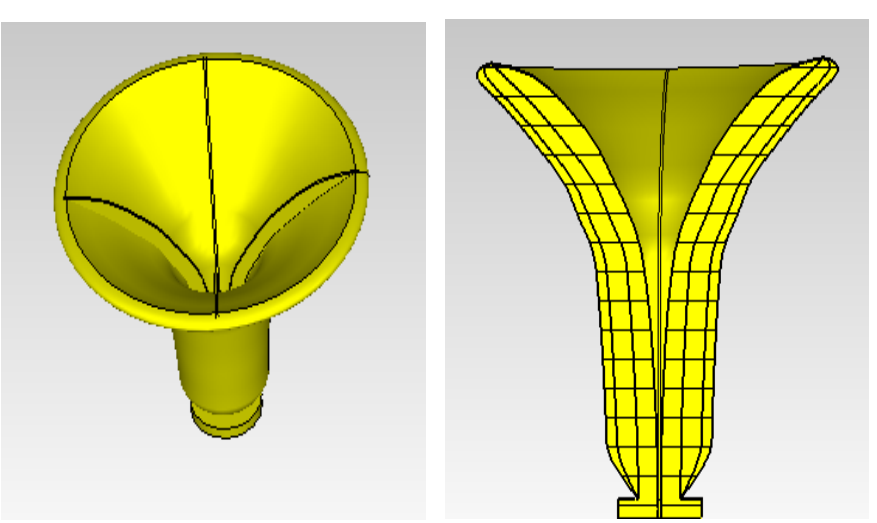

Fig. 1. Geometry of the new spline-profiled quadruple-ridge flared horn (QRFH).

SKA WBSPF Band B (4.6-24 GHz).

\section{STRUCTURE}

It was found that the existing analytical profiles for ridge and horn in QRFHs, such as elliptic, exponential, power of variables, power of tangential, power of hyperbolic, power of polynomial, power of asymmetric sine-squared, and their combinations [2], [3], could not meet the specification of SKA Band B. In addition, all previous developed QRFHs have thin ridges in terms of the wavelength, which makes the ridges too thin for frequencies up to $24 \mathrm{GHz}$ to manufacture in a suitable way. Through a study of large amounts of simulations, it can be concluded that the ridge profile controls the beamwidth in E-plane while the horn profile has more influence on the beamwidth in H-plane, and the performance of QRFH is quite sensitive to the profile shape of the ridges and horn. Therefore, more dedicated effort on optimizing these profiles is needed. The new solution of spline-profiled QRFH has ridges and horn with numerically defined profiles which control the propagation modes in QRFH to maximize the aperture efficiency. 


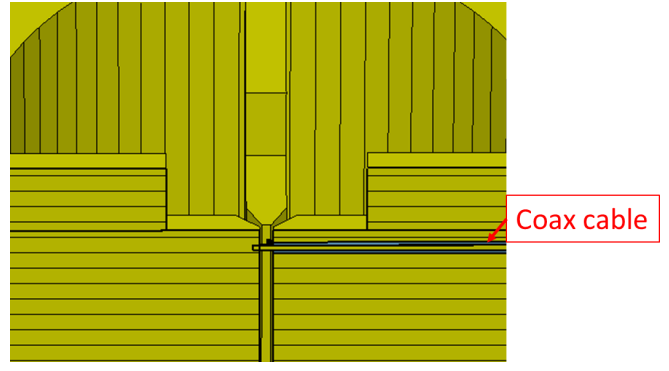

Fig. 2. Structure of coax-to-ridge transition.

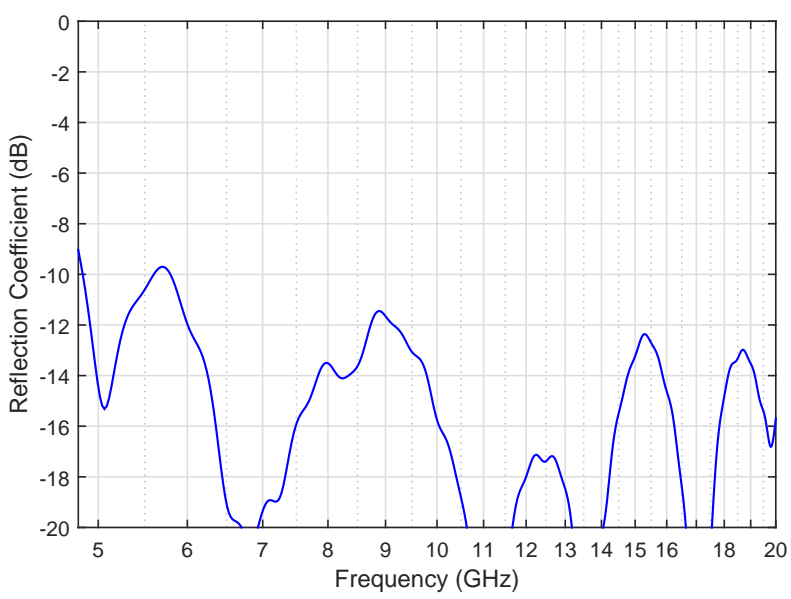

Fig. 3. Simulated reflection coefficient including both the QRFH part and coax-to-ridge transition part.

The spline-profile means that the spline curve function in CST is used to make the profile for ridges, horn and coaxto-ridge transition. This technique gives advantages in both flexibility and smoothness of the profiles. Both the ridge and horn profiles are defined by seven points and the transition is defined by three points. All these points are optimized numerically to get the maximum aperture efficiency and minimum reflection coefficient at the same time.

The design procedure has a few steps. First, the QRFH and the coax-to-ridge transition part are optimized individually. Fig. 1 shows the optimized QRFH part, and Fig. 2 shows the transition part. Then, the whole antenna with both QRFH and transition is optimized further. The whole antenna is about $170 \mathrm{~mm}$ in diameter and $170 \mathrm{~mm}$ in length.

\section{Simulated Results}

Fig. 3 shows the simulated reflection coefficient of the whole QRFH antenna (both QRFH part and coax-to-ridge transition included) below $-10 \mathrm{~dB}$ over $4.6-20 \mathrm{GHz}$. The simulated aperture efficiency when the QRFH antenna feeds the SKA dual reflector (shown in Fig. 4(a)) is presented in Fig. 4(b), which is above $70 \%$ up to $16.5 \mathrm{GHz}$ and above $65 \%$ up to $20 \mathrm{GHz}$. Note that we are still optimizing the performance over 20-24 GHz.

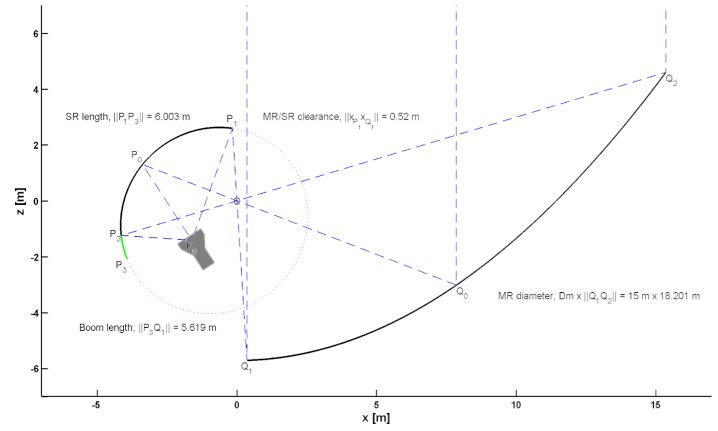

(a)

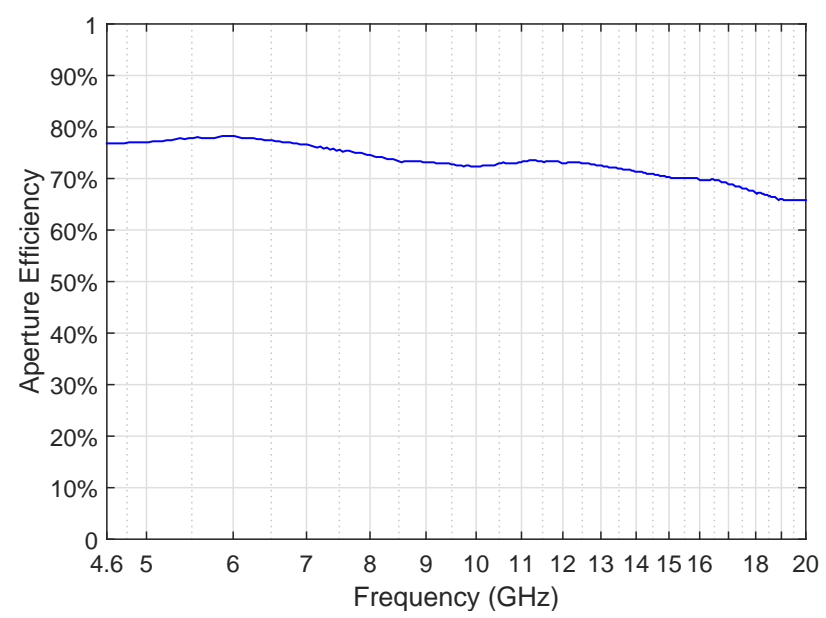

(b)

Fig. 4. (a) SKA reflector geometry, an offset Gregorian dual reflector with half subtended angle $\theta_{e}=58^{\circ}$; (b) Simulated aperture efficiency of the 4.6$20 \mathrm{GHz}$ feed in the SKA reflector.

\section{CONCLUSIONS}

A new quadruple-ridge flared horn with spline-defined profile as a feed for Square Kilometer Array Band B is proposed in the paper. The simulated reflection coefficient and aperture efficiency are below $-10 \mathrm{~dB}$ and above $60 \%$ over $4.6-20 \mathrm{GHz}$, respectively.

\section{ACKNOWLEDGEMENT}

This work has been supported by Swedish VR Research Infrastructures Planning Grant Swedish contributions to the SKA radio-telescope in its pre-construction phase (Contract No. C0546801).

\section{REFERENCES}

[1] I. P. Theron, R. Lehmensiek, and D. de Villiers, "Towards an optics design for SKA," in IEEE AFRICON, , Mauritius, Sep. IEEE, 2013.

[2] A. Akgiray, S. Weinreb, W. Imbriale, and C. Beaudoin, "Circular quadruple-ridged flared horn achieving near-constant beamwidth over multioctave bandwidth: Design and measurements," IEEE Trans on Antennas and Propagat., vol. 61, no. 3, pp. 1099-1108, March 2013.

[3] M. Ivashina, R. Bradley, M. Pantaleev, J. Yang, and C.Bencivenni, "Ultrawideband, cryogenically-cooled reflector antenna feeds for future radio telescopes: Conical-sinuous antenna, Eleven antenna and quad-ridged flared horn feeds," in Proceedings of the 2014 XXXIth URSI General Assembly and Scientific Symposium (URSI GASS), Beijing, China, Aug. URSI, 2014. 